\title{
树冠结构对典型阔叶红松林生产力的影响
}

\author{
哈努拉・塔斯肯 ${ }^{1}$ 蔡慧颖 2,3 金光泽 ${ }^{1,3 *}$ \\ ${ }^{1}$ 东北林业大学生态研究中心, 哈尔滨 $150040 ;{ }^{2}$ 东北林业大学林学院, 哈尔滨 $150040 ;{ }^{3}$ 东北林业大学森林生态系统可持续经营教育部重点实验室, \\ 哈尔滨 150040
}

摘 要 阔叶红松(Pinus koraiensis)林是东北东部山区的地带性森林植被, 阐明其生产力的影响因素, 对于理解温带森林生 产力维持机制具有重要意义。该研究依托小兴安岭典型阔叶红松林 $9 \mathrm{hm}^{2}$ 动态监测样地，基于 2005 和 2015 年的 $30 \mathrm{~m} \times 30 \mathrm{~m}$ 样 方内所有胸径 $>6.5 \mathrm{~cm}$ 的木本植物的调查数据, 计算各样方的树冠结构复杂性、物种多样性和林分胸高断面积, 结合各样方的 地形和土壤理化性质数据, 拟合结构方程模型, 定量分析影响典型阔叶红松林生产力的直接和间接因素。研究结果显示: 树 冠结构复杂性和物种多样性与生产力显著正相关, 且树冠结构复杂性对生产力的影响显著高于物种多样性; 树冠结构复杂性 对生产力的作用分为树冠垂直分层和树冠可塑性, 其中树冠垂直分层是树冠结构复杂性影响阔叶红松林生产力的主要因素, 而树冠可塑性无显著影响; 林分胸高断面积与生产力显著正相关, 其解释权重仅次于树冠结构复杂性, 树冠结构复杂性与物 种多样性均通过影响林分胸高断面积对阔叶红松林生产力产生间接影响; 考虑不同树冠结构复杂性时, 坡度和土壤全磷含量 代表的环境因素在调节生产力上发挥的作用存在差异, 移除树冠垂直分层的作用后两者与生产力呈显著的负相关关系。综上 可知, 在典型阔叶红松林中, 树冠结构复杂性比物种多样性更有效地解释了生产力的变化, 同时不可忽视其他生物和非生物 因素对生产力的作用。

关键词 生物多样性; 树冠结构复杂性; 物种均匀度; 生产力; 阔叶红松林

哈努拉·塔斯肯, 蔡慧颖, 金光泽 (2021). 树冠结构对典型阔叶红松林生产力的影响. 植物生态学报, 45, 38-50. DOI: 10.17521/cjpe.2020.0176

\section{Effects of canopy structure on productivity in a typical mixed broadleaved-Korean pine forest} Hanula TASIKEN ${ }^{1}$, CAI Hui-Ying ${ }^{2,3}$, and JIN Guang-Ze ${ }^{1,3^{*}}$

${ }^{1}$ Center for Ecological Research, Northeast Forestry University, Harbin 150040, China; ${ }^{2}$ School of Forestry, Northeast Forestry University, Harbin 150040, China; and ${ }^{3}$ Key Laboratory of Sustainable Forest Ecosystem Management Ministry of Education, Northeast Forestry University, Harbin 150040, China

\section{Abstract}

Aims The mixed broadleaved-Korean pine (Pinus koraiensis) forest is a zonal vegetation type in Northeast China. Clarifying the influencing factors of productivity is of great significance for understanding the maintenance mechanism of productivity in temperate forests.

Methods The study was conducted based on survey data of a $9 \mathrm{hm}^{2}$ permanent plot in a typical mixed broadleavedKorean pine forest in the Xiaoxing'an Mountains. Census data of 2005 and 2015 for all individuals with diameter at breast height $>6.5 \mathrm{~cm}$ within each $30 \mathrm{~m} \times 30 \mathrm{~m}$ sub-plot were used to calculate canopy structural complexity, species diversity and stand basal area. We analyzed the direct and indirect factors affecting forest productivity using structural equation model and data on topography and soil physicochemical properties in each sub-plot.

Important findings Both the canopy structural complexity and the species diversity were positively related to productivity, with canopy structural complexity having a significantly greater effect than species diversity. The effects of canopy structural complexity on productivity consisted of two attributes: the vertical stratification and the plasticity. The vertical stratification played a significant role in driving the changes in forest productivity, while plasticity had no significant effect. The stand basal area was positively related to productivity, but with less effect than the canopy structural complexity. Both the canopy structural complexity and the species diversity had indirect effects on productivity via stand basal area. However, the environmental factor representative of slope and soil total phosphorus played differential roles on productivity for varying canopy structral complexity, and it was significantly and negatively correlated with productivity after removing the effects of canopy vertical stratification. In short, in a typical mixed broadleaved-Korean pine forest, the canopy structural complexity is more crucial for

收稿日期Received: 2020-05-29 接受日期Accepted: 2020-08-28

基金项目：国家自然科学基金(31901299)。Supported by the National Natural Science Foundation of China (31901299).

* 通信作者Corresponding author (taxus@126.com) 
explaining the diversity-productivity relationship than species diversity, and the roles of other biotic and abiotic factors in productivity should not be ignored.

Key words biodiversity; canopy structural complexity; species evenness; productivity; mixed broadleavedKorean pine forest

Tasiken H, Cai HY, Jin GZ (2021). Effects of canopy structure on productivity in a typical mixed broadleaved-Korean pine forest. Chinese Journal of Plant Ecology, 45, 38-50. DOI: 10.17521/cjpe.2020.0176

生物多样性-生产力关系(BPR)是理解全球物种 灭绝危机及其对生态系统功能影响的基础, 对于准 确评估和有效保护生物多样性至关重要(Zhang et al., 2012; Liang et al., 2016; Yuan et al., 2018)。早期 的BPR研究多集中于分析物种多样性的作用, 对生 产力的解释和预测能力较低 (Grace et al., 2016; Zeller et al., 2018)。近年来, 随着对生物多样性不同 维度(物种、功能、谱系和结构多样性)认识的加深 和扩展, 生态学家们基于树冠结构在垂直和水平方 向上的差异提出林分结构多样性的衍生多样性— 树冠结构复杂性。树冠结构复杂性作为综合评价森 林冠层对光照时空分配利用情况的重要参数, 可以 较全面地描述森林的林分结构特征(Sapijanskas et al., 2014), 其值越高说明林内个体对立地空间的利用 率越高, 对林分中光照等资源的利用更全面, 进而 具有更高的森林生产力(Ali et al., 2019)。

树冠结构复杂性可分两部分来分别解释树冠结 构在垂直与水平方向上的作用(Pretzsch, 2014; Jucker et al., 2015)。一是树冠垂直分层: 当树木的叶片分 布在冠层的不同高度剖面时, 森林冠层能够最大限 度地截留光线, 从而对森林生产力产生积极的促进 作用(Williams et al., 2017)。共存树种对光照的分层 截留以及对郁闭环境的生理适应, 均有利于树冠垂 直分层(Sterba et al., 2019)。二是树冠可塑性：树木 的大小和形状在生长过程中具有较强的可塑性, 可 以形成镶嵌的树冠结构, 覆盖大部分或所有的树冠 空间(Purves et al., 2007)。树冠横向发展, 如展叶、 分枝和树冠形状的变化, 均有助于减少林分中激烈 的种内、种间竞争, 进而促进森林生态系统功能优 化(Jucker et al., 2015; Fotis et al., 2018)。树冠结构复 杂性被系统地提出之后, 该研究领域的成果迅速增 加, 大量实验证明树冠结构复杂性可以提高森林生 态系统的生产力, 但是树冠垂直分层和树冠可塑性 二者的作用强度和内在作用机制还有争议(Pretzsch, 2014; Fahey et al., 2015)。部分学者认为多林层林分 具有更高的生产潜能, 林冠垂直分层是诸多森林生
物量积累的重要驱动因素(Hardiman et al., 2013; Dănescu et al., 2016)。然而, 这与Bayer等(2013)和 Jucker等(2015)的研究相悖, 他们通过分离垂直和 水平方向上的树冠结构复杂性发现, 可塑性较强的 树冠结构才是影响生产力的重要因素, 树冠垂直分 层反而没有明显作用。不同地区和林龄中树冠结构 复杂性对生产力的贡献相异(Bohn \& Huth, 2017), 因此仍需更多系统的研究来阐明树冠垂直分层和树 冠可塑性的相对重要性。此外, 林分中较高的物种 丰富度, 往往会形成较高的树冠结构复杂性, 从而 间接地促进森林生产力。然而, 物种多样性对生产 力的这种间接影响尚未得到完全证实(Forrester, 2014; Sapijanskas et al., 2014)。

通常, 树木生长是多种因素共同作用的结果, 在研究多样性-生产力关系时考虑气候、地形、土壤 养分和群落结构等多个生物和非生物因素的作用十 分必要, 有利于更好地理解多驱动因素对天然林多 样性和生态系统功能的影响(Ali et al., 2016; Yuan et al., 2018)。区域尺度上, 林分胸高断面积是影响 生产力的重要因子。多数研究以单位面积内所有个 体的胸高断面积之和作为木本植物地上生物量的衡 量指标, 探讨其与生产力的相关关系(郭屹立等, 2016)。林分胸高断面积越大, 森林生产潜力越高 (Muth \& Bazzaz, 2003)。此外, 物种多样性和树冠结 构也会通过影响林分胸高断面积, 间接地驱动森林 生产力(Dănescu et al., 2016)。地形与土壤物理化学 属性互相联系、互相制约, 是导致不同尺度环境异 质性的重要因素, 地形的变化可以引起水、肥、气、 热的重新分配, 进而影响植物的分布和生长(Williams et al., 2017)。如通常在地势平坦地区, 土壤有机质 和土壤水分较高, 树木生长速度较快, 对森林生产 力存在正向的影响(Zheng et al., 2019); 但较高的土 壤养分可利用性，也会加剧种内、种间的竞争，导致 树木较高的死亡率和周转率(Quesada et al., 2012)。 综上, 生物因素和非生物因素对生产力的影响仍需 要进一步验证。 
阔叶红松(Pinus koraiensis)林主要分布于我国 东北东部山区, 是该地区典型的地带性顶极植被。 与全球同纬度地区的森林相比, 阔叶红松林拥有独 特的建群种、丰富的物种多样性以及复杂的森林群 落结构, 在水土保持、环境保护以及生物多样性维 持等方面发挥着重要作用。然而由于过去几十年的 过度采伐, 目前仅有少部分原始阔叶红松林分布于 保护区和国家公园等地区(王业遁, 1995)。基于以上 背景, 本研究依托黑龙江凉水国家级自然保护区9 $\mathrm{hm}^{2}$ 润叶红松林动态监测样地, 利用结构方程模型, 分析树冠结构复杂性、物种多样性、林分胸高断面 积和环境因素对阔叶红松林生产力的影响, 试图回 答以下问题: (1)在阔叶红松林中, 树冠结构复杂性 和物种多样性是否能够独立地影响生产力的变化? 二者作用强度如何? (2)树冠结构复杂性对生产力的 影响主要是垂直分层的作用还是树冠可塑性的作用? (3)其他生物因素和非生物因素是否对阔叶红松林 生产力产生影响?

\section{1 材料和方法}

\section{1 研究样地概况}

本研究在黑龙江凉水国家级自然保护区进行, 保护区隶属黑龙江省伊春市大等山县, 处小兴安岭 南部达里带岭支脉东坡 $\left(47.16^{\circ} \mathrm{N}, 128.95^{\circ} \mathrm{E}\right.$, 海拔 280-707 m)。保护区内森林植被覆盖率高达96\%, 是 我国现有为数不多的大面积原始阔叶红松林分布区 之一。研究区位于欧亚大陆东侧, 深受海洋气候影 响, 具有明显的温带大陆性季风气候特征。研究区 年平均气温 $-0.3{ }^{\circ} \mathrm{C}$, 极端最高气温 $38.2{ }^{\circ} \mathrm{C}$, 极端最 低气温-43.1 ${ }^{\circ} \mathrm{C}$; 年降水量 $676 \mathrm{~mm}$, 主要集中在 6-9月 (占76\%), 雨热同季。年平均相对湿度介于 $78 \%-96 \%$ 之间, 年蒸发量 $805.4 \mathrm{~mm}$; 年日照时间 $1850 \mathrm{~h}$, 无霜期100-120天(https://ls.nefu.edu.cn/)。 地带性土壤为暗棕壤, 非地带性土壤为草甸土、沼 泽土和泥炭土。全区植被以红松为优势种, 伴生有 紫椴(Tilia amurensis)、糠椴(Tilia mandshurica)、五 角枫(Acer pictum subsp. mono)、青楷槭(Acer tegmentosum)、花楷槭(Acer ukurunduense)、裂叶榆 (Ulmus laciniata)和水曲柳(Fraxinus mandshurica) 等 20 余种针阔叶树种; 灌木有刺五加(Eleutherococcus senticosus)、毛榛(Corylus mandshurica) 和东北山梅花
(Philadelphus schrenkii)等(王业茩, 1995)。

\section{2 样地设置与数据调查}

2005年, 参照BCI (Barro Colorado Island) $50 \mathrm{hm}^{2}$ 样地的操作技术规范, 在黑龙江凉水国家级自然保 护区内建立了 $9 \mathrm{hm}^{2}(300 \mathrm{~m} \times 300 \mathrm{~m})$ 阔叶红松林动 态监测样地(徐丽娜和金光泽, 2012)。将整个样地划 分成 900 个 $10 \mathrm{~m} \times 10 \mathrm{~m}$ 的样方, 逐个样方进行调查, 精确测量样方内所有胸径 $\geqslant 2 \mathrm{~cm}$ 的木本植物, 记录 每株树木的种名、胸径、树高、坐标和生活状态(存 活、倒伏、枯立)并挂牌; 2007年使用Vertex III超声 波测高仪(Haglof, Langsele, Sweden)对样地内所有 挂牌活立木的树高和树枝下高进行测量, 并对树高 $>10 \mathrm{~m}$ 的个体进行冠幅调查: 以树干为中心测量 8 个 方向(即东、西、南、北、东南、西北、东北和西 南方向)的冠幅长度; 分别于 2010 和 2015 年对胸径 $\geqslant 2 \mathrm{~cm}$ 的木本植物进行了调查。本研究采用 2005-2015年10年间隔的森林动态监测数据计算森 林生产力。

本文参考有关树冠结构复杂性的研究, 采用胸 径 $>6.5 \mathrm{~cm}$ 的个体进行计算, 即舍弃复查期间容易 消失的小径级树木, 保证目标树处于冠层, 使结果 更具生态学意义(Fahey et al., 2015; Jucker et al., 2015)。本研究预分析了不同尺度下树冠结构复杂性 和物种多样性与生产力关系(附录I), 其中 $30 \mathrm{~m} \times 30$ $\mathrm{m}$ 尺度下模型赤池信息准则 $(A I C)$ 最低, 故主要研究 $30 \mathrm{~m} \times 30 \mathrm{~m}$ 空间尺度下的多样性-生产力关系。

\section{3 数据分析}

\subsection{1 群落木本生产力的计算}

根据东北主要林木生物量的异速生长方程(陈 传国和朱俊风，1989), 利用胸径计算木本植株的地 上生物量。参考Chave等(2003)的方法, 计算阔叶红 松林群落木本生产力 $(C W P)$ 。计算公式为:

$$
C W P=\frac{G+R}{0.09 \times T}
$$

式中, $G$ 为两次调查时存活个体的生物量增量; $R$ 为 样方内 2005 年调查时胸径 $<6.5 \mathrm{~cm}, 2015$ 年复查时胸 径 $>6.5 \mathrm{~cm}$ 的新增个体的生物量总和; 样方面积为 $0.09 \mathrm{hm}^{2} ; T$ 为 10 年。

\subsection{2 树冠体积的计算}

根据2007年的冠幅调查数据, 针对胸径 $>6.5 \mathrm{~cm}$ 的冠层树木, 分别计算东、南、西、北方向和东北、 
西南、东南、西北方向的冠幅总长度, 利用两者中 相对较大的冠幅总长度的平均值作为最大的树冠半 径, 结合每株树的树高和枝下高, 计算每株个体的 树冠体积。计算公式为:

$$
C V=\int_{H-C D}^{H} \pi(f(h))^{2} \mathrm{~d} h
$$

式中, $C V$ 为树冠体积, $H$ 为树高, $H-C D$ 为枝下高, $h$ 为 某处的冠层高度, $\mathrm{d} h$ 为 $h$ 的导数。

为了准确地估算树冠体积, 将树冠体积视为抛 物线锥, 化简积分加入代表树冠形状的参数 $\beta$ 。 $\beta$ 值 决定树冠的形状, $\beta=0$ 时该抛物线雉体更接近圆柱 体, $\beta=1$ 时为圆雉体。从文献中获得针叶树种和阔 叶树种树冠形状参数的平均 $\beta$ 值分别为 0.26 和 0.44 , 使得针叶和阔叶树种的树冠体积曲率更贴合抛物线 雉体(图1)(Purves et al., 2007)。化简方程为:

$$
C V=\frac{\pi C R_{\max }^{2} C D}{2 \beta+1}
$$

式中, $C D$ 为树冠高度, $C R_{\max }$ 为最大树冠半径, $\beta$ 为冠 形参数。

\subsection{3 树冠结构复杂性的计算}

树冠结构复杂性是由冠层镶嵌指数测算, 冠层 镶嵌指数被定义为树木潜在的可利用地上空间的比 例，而这些空间实际由冠层占据(Jucker et al., 2015)。

$$
C P=\frac{\sum C V}{\text { plotarea } \times\left[H_{\max }-(H-C D)_{\min j}\right]}
$$

式中, $C P$ 为冠层镶嵌指数; $\Sigma C V$ 代表样方内所有立

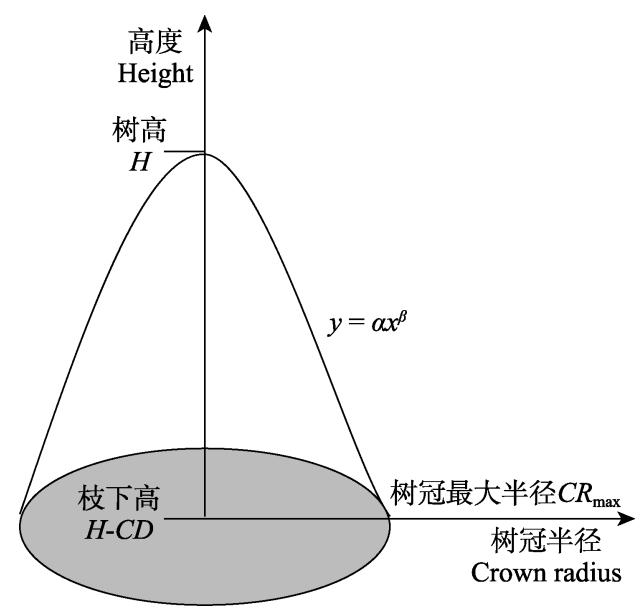

图1 树冠体积的示意图。 $y=\alpha x^{\beta}$ 表示树冠形状拟合的抛物 线公式, $\beta$ 为冠形参数。

Fig. 1 Schematic illustration of crown volume of a tree. $C R_{\max }$, the maximum of radius of crown; $H$, tree height; $H-C D$, under branch height. The shape of the crown is determined by equation $y=\alpha x^{\beta}$, and $\beta$ is the shape parameter which determines the curvature of the crown.
木的树冠体积之和; plot area为样方面积, 本研究中 为 $0.09 \mathrm{hm}^{2} ; H_{\operatorname{maxj}}$ 为 $j$ 样方内单木树高最大值, $(H-C D)_{\min j}$ 为 $j$ 样方内单木枝下高最小值。式中的分 子代表树木在样方内实际占据的空间，分母则表示 树木在样方中可能占据的所有空间。冠层镶嵌指数 为一个比例，无单位，取值范围在0-1之间, 0 表示无 冠层填充, 1 表示冠层完全郁闭。

树冠垂直分层的作用也是由冠层镶嵌指数计算 所得, 其中 $\sum C V$ 是由每一株树木的树冠体积异速 生长方程计算, 旨在去除个体树冠体积中与树木大 小无关的种内变异的部分, 即移去树冠可塑性的作 用。计算具体如下：对每个物种的 $\log (C V)$ 和 $\log$ $(D B H)$ 拟合线性回归模型，采用最小二乘法检验回 归模型; 利用各个树种的树冠体积异速生长方程计 算每株树的树冠体积, 纳入公式(4)中计算样方中树 冠垂直分层所占的比例。树冠可塑性，是表征单木 树冠在林分中横向扩张程度的指标, 代表树冠水平 方向上的异质程度, 其值为树冠结构复杂性与垂直 分层比例的差值。

\subsection{4 物种多样性的计算}

在 $30 \mathrm{~m} \times 30 \mathrm{~m}$ 样地空间尺度上, 计算各样方中 物种多样性, 具体采用 Shannon多样性指数 $\left(H^{\prime}\right)$ 、 Simpson多样性指数 $\left(D^{\prime}\right)$ 、均匀度指数 $\left(E^{\prime}\right)$ 和物种丰 富度指数 $(S)$ 代表物种多样性(表1), 计算公式如下:

Shannon多样性指数: $H^{\prime}=-\sum_{i=1}^{N_{\mathrm{s}}} \frac{n_{i}}{N} \times \ln \left(\frac{n_{i}}{N}\right)$

Simpson多样性指数: $D^{\prime}=1-\sum_{i=1}^{N_{\mathrm{s}}}\left(\frac{n_{i}}{N}\right)$

均匀度指数: $E^{\prime}=\frac{D}{N_{\mathrm{s}}}$

物种丰富度指数: $S=N_{\mathrm{s}}$

式中, $N$ 为样方内总个体数; $N_{\mathrm{s}}$ 为样方内物种总数; $n_{i}$ 为第 $i$ 个物种的个体数。

\subsection{5 环境因素的计算}

本研究中环境因素包括地形和土壤理化性质。 地形变量包括海拔、坡向、坡度和凹凸度。将 $9 \mathrm{hm}^{2}$ 阔叶红松林动态监测样地划分为 100 个 $30 \mathrm{~m} \times 30 \mathrm{~m}$ 小样方, 样地内所有地形变量均根据每个小样方单 元四个角的高度计算(表2)。此外, 由于坡向为 $0^{\circ}-360^{\circ}$ 的数值, 在分析和建模时不能直接使用, 需 要进行余弦和正弦函数转换, 分别代表小样方偏北 向和东向的程度(Legendre et al., 2009)。 
表1 典型阔叶红松林样地物种多样性指数统计表

Table 1 Summary of species diversity index in a typical mixed broadleaved-Korean pine forest plot

\begin{tabular}{lccc}
\hline 变量 Variable & 平均值 Mean & 标准差 $S D$ & 范围 Range \\
\hline Shannon多样性指数 Shannon diversity index & 0.41 & 0.30 & $0.12-1.50$ \\
Simpson多样性指数 Simpson diversity index & 0.18 & 0.12 & $0.06-0.54$ \\
均匀度指数 Evenness index & 0.15 & 0.12 & $0.04-0.58$ \\
物种丰富度指数 Species richness index & 9.48 & 1.99 & $5-16$ \\
\hline
\end{tabular}

$S D$, standard deviation.

表2 典型阔叶红松林样地地形和土壤物理化学性质状况统计表

Table 2 Summary of topographic factors and soil physical and chemical properties in a typical mixed broadleaved-Korean pine forest plot

\begin{tabular}{|c|c|c|c|c|}
\hline 环境因子 Environmental factor & 平均值 Mean & 标准差 $S D$ & 变异系数 $C V$ & 范围 Range \\
\hline 海拔 Elevation (m) & 463.22 & 18.07 & 0.04 & $427.93-499.07$ \\
\hline 坡度 Slope $\left(^{\circ}\right)$ & 15.30 & 4.67 & 0.31 & $8.10-33.40$ \\
\hline 坡向 Aspect (sin) & 0.02 & 0.72 & 36.00 & $-0.997-0.994$ \\
\hline 坡向 Aspect (cos) & -0.07 & 0.70 & -10.00 & $-0.999-0.999$ \\
\hline 凹凸度 Convex & 0.06 & 1.05 & 17.50 & $-1.55-4.45$ \\
\hline 土壤速效磷含量 Soil available phosphorus content $\left(\mathrm{mg} \cdot \mathrm{kg}^{-1}\right)$ & 8.83 & 6.04 & 0.68 & $3.53-43.11$ \\
\hline 土壤速效钾含量 Soil available potassium content $\left(\mathrm{mg} \cdot \mathrm{kg}^{-1}\right)$ & 332.61 & 52.69 & 0.16 & $200.87-485.34$ \\
\hline 土壤速效氮含量 Soil available nitrogen content $\left(\mathrm{mg} \cdot \mathrm{kg}^{-1}\right)$ & 1103.60 & 147.95 & 0.13 & $666.56-1450.17$ \\
\hline 土壤有机碳含量 Soil organic carbon content $\left(\mathrm{g} \cdot \mathrm{kg}^{-1}\right)$ & 75.75 & 29.71 & 0.39 & $37.63-186.29$ \\
\hline 土壤全氮含量 Soil total nitrogen content $\left(\mathrm{g} \cdot \mathrm{kg}^{-1}\right)$ & 8.40 & 1.46 & 0.17 & $5.22-11.70$ \\
\hline 土壤全磷含量 Soil total phosphorus content $\left(\mathrm{g} \cdot \mathrm{kg}^{-1}\right)$ & 0.82 & 0.13 & 0.16 & $0.44-1.13$ \\
\hline 土壤pH Soil pH & 5.77 & 0.15 & 0.03 & $5.47-6.19$ \\
\hline 土壤容重 Soil bulk density $\left(\mathrm{g} \cdot \mathrm{cm}^{-3}\right)$ & 0.62 & 0.09 & 0.15 & $0.43-0.85$ \\
\hline 体积含水率 Soil volumetric moisture content (\%) & 30.35 & 5.37 & 0.18 & $15.44-44.06$ \\
\hline 质量含水率 Soil mass moisture content $\left(\mathrm{g} \cdot \mathrm{g}^{-1}\right)$ & 0.92 & 0.22 & 0.24 & $0.55-1.40$ \\
\hline
\end{tabular}

$C V$, coefficient of variation; $S D$, standard deviation.

在 $20 \mathrm{~m} \times 20 \mathrm{~m}$ 小样方原点位置, 取1个样点, 同时随机选择一个方向，在离原点 $2 、 5$ 和 $8 \mathrm{~m}$ 处选任 意 2 个样点, 取土壤表层 $(0-10 \mathrm{~cm})$ 的样品, 于实验 室内测定土壤容重 $(B D)$ 、土壤 $\mathrm{pH}$ 、土壤速效钾含量 $(A K)$ 、土壤速效磷含量 $(A P)$ 、土壤有效氮含量 $(A N)$ 、 质量含水率 $(M M)$ 、土壤有机质含量 $(O C)$ 、土壤全氮 含量 $(T N)$ 、土壤全磷含量 $(T P)$ 和体积含水率 $(V M)$ (Klute, 1986)。将每个 $20 \mathrm{~m} \times 20 \mathrm{~m}$ 的小样方分 为 16 个 $5 \mathrm{~m} \times 5 \mathrm{~m}$ 的子样方后, 利用A ArcGIS 10.1软件 地统计学分析工具中的ordinary Kriging方法对土壤 理化性质的 10 项指标进行空间插值，从而获得 $30 \mathrm{~m}$ $\times 30 \mathrm{~m}$ 取样单元的基础环境变量指标(表2)。

\subsection{6 统计分析}

本文利用结构方程模型, 综合分析树冠结构复 杂性、物种多样性、林分胸高断面积和环境因素与 典型阔叶红松林生产力之间的多元关系。根据多样 性-生产力关系, 在已知理论的基础上构建初始模 型(图2)(Ali et al., 2016; Zheng et al., 2019)。模型中

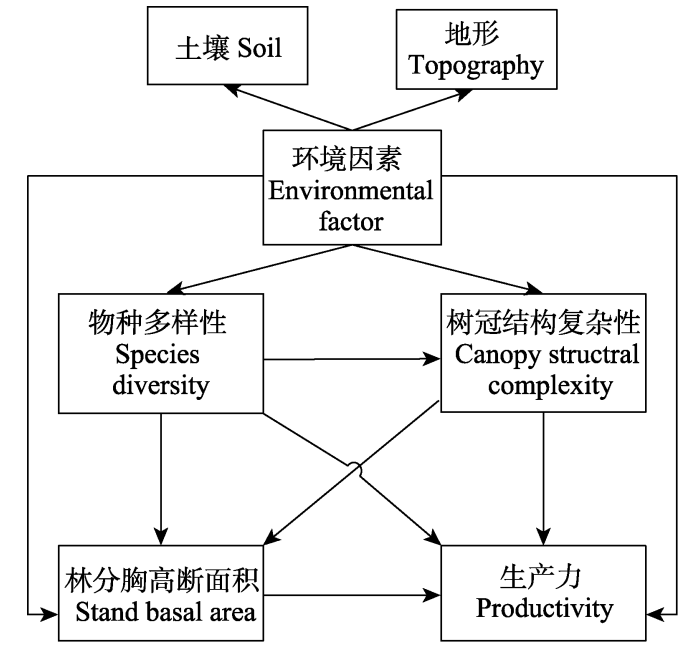

图2 阔叶红松林环境因素、物种多样性、树冠结构复杂性、 林分胸高断面积与生产力之间多元关系的概念模型。其中环 境因素为潜变量, 包括土壤理化性质和地形因子。

Fig. 2 A conceptual model of hypothesized causal pathways linking the environmental factor, species diversity, canopy structural complexity, stand basal area, and productivity in a mixed broadleaved-Korean pine forest. The environmental factor is a latent variable consisting of soil physical and chemical properties and topography. 
不仅考虑了环境因素对物种多样性、树冠结构复杂 性和生产力的影响, 而且还考虑了林分胸高断面积 与环境因素、物种多样性、树冠结构复杂性、林分 生产力之间的关系(Muth \& Bazzaz, 2003)。

为了使响应变量呈正态分布, 对生产力进行对 数转化。用 $Z$-score标准分数对解释变量进行标准化 处理, 将不同量级的解释变量转化为同一量级, 以 保证变量间标准系数的可比性。根据各个因子与生 产力多重回归模型的结果, 分别篮选出重要性最 高、与生产力最为相关的多样性指数和环境变量, 纳入结构方程模型中(附录II)。根据变量篮选结果, 最终以均匀度指数代表物种多样性, 土壤全磷含量 及坡度组成的潜变量代表环境变量, 纳入考虑树冠 结构复杂性、树冠垂直分层和树冠可塑性的 3 个结构 方程模型中, 获得解释阔叶红松林生产力的最优模 型(表3; 图3)。

本文选择最大似然法对结构方程模型进行拟合, 利用比较拟合指数 $(C F I)$ 、标准均方根残差 $(S R M R)$ 、 渐进残差均方和平方根 $(R M S E A)$ 和显著性概率值 $(p)$ 对模型进行拟合优度评价。相应的拟合优度的临界 值为 $C F I>0.9 、 S R M R<0.05 、 R M S E A<0.08$ 和 $p>$ 0.05 (Howard, 2013)。采用R 3.6.0进行数据分析, 变 量篮选在MuMIn包中进行(Barton, 2016), 结构方程 模型的运算分析利用lavaan包完成(Rosseel，2012), 使用Microsoft Visio 2010软件和SigmaPlot 10.0软件 绘图。

\section{2 结果}

\section{1 树冠结构复杂性对典型阔叶红松林生产力的} 作用

在考虑树冠结构复杂性的模型中, 各个变量解 释典型阔叶红松林生产力变异的 $34.9 \%$ 。树冠结构 复杂性和物种多样性对生产力的直接效应分别为 $0.229(p=0.045)$ 和 $0.284(p=0.006)$, 通过林分胸高
断面积间接地作用于生产力的间接效应分别为 $0.227(p=0.004)$ 和 $-0.158(p=0.008)$, 两者的总效 应(直接和间接效应之和)分别为 $0.455(p<0.001)$ 和 $0.207(p=0.036)$, 树冠结构复杂性与生产力的关系 比物种多样性更密切(图3A; 表4)。林分胸高断面积 对生产力的直接效应为 $0.379(p=0.002)$, 而土壤全 磷和坡度结合的环境因素对生产力无显著的直接影 响 $(p>0.05)$, 通过林分胸高断面积的间接效应为 $0.137(p=0.021)$ 。物种多样性与树冠结构复杂性呈 极显著正相关关系, 路径系数为0.357 $(p<0.001)$, 但通过树冠结构作用于生产力的间接影响不显著 $(p$ $>0.05)$ (图3A; 表4)。

\section{2 树冠垂直分层对典型阔叶红松林生产力的作用}

在仅考虑树冠垂直分层的模型中, 各个变量解 释阔叶红松林生产力变异的 $42.0 \%$, 在 3 个模型中对 生产力变异解释度最高。在此模型中, 树冠垂直分 层对阔叶红松林生产力的直接效应为 $0.434(p<$ $0.001)$, 总效应为 $0.560(p<0.001)$, 是各个变量中 最重要的解释因子; 物种多样性和生产力的相关关 系相对较低, 直接效应为 $0.244(p=0.007)$, 总效应 为 $0.263(p=0.006)$; 物种多样性与树冠垂直分层无 显著的相关关系 $(p>0.05)$, 表明二者独立地影响生 产力。环境因素和林分胸高断面积与生产力无显著 的相关关系 $(p>0.05)$ (图3B; 表5)。

\section{3 树冠可塑性对典型阔叶红松林生产力的作用}

在仅考虑树冠可塑性的模型中, 各个变量解释 阔叶红松林生产力变异的 $32.2 \%$, 相对于其他两个 模型解释程度较低。在此模型中, 林分胸高断面积 是各个变量中最重要的解释因子, 其直接效应(总 效应)为 $0.544(p<0.001)$; 其次为物种多样性, 其直 接效应为 $0.417(p<0.001)$, 通过林分胸高断面积对 生产力产生的间接效应为 $-0.178(p=0.005)$, 总效 应为 $0.222(p=0.030)$; 环境因素对生产力的直接效 应为-0.264 ( $p=0.024)$, 且通过林分胸高断面积间

表3 典型阔叶红松林最优结构方程模型检验多样性与生产力关系的结果

Table 3 Results of the best-fit structural equation models (SEMs) testing the relationships between forest productivity and diversity in a typical mixed broadleaved-Korean pine forest

\begin{tabular}{lcccc}
\hline 模型 Model & $C F I$ & SRMR & RMSEA & AIC \\
\hline 树冠结构复杂性 Canopy structural complexity & 1 & 0.020 & 0 & 1308.25 \\
树冠垂直分层 Canopy vertical stratification & 1 & 0.019 & 0.720 & 1295.63 \\
树冠可塑性 Canopy plasticity & 1 & 0.021 & 0 & 1346.06 \\
\hline
\end{tabular}

$A I C$, 赤池信息准则; $C F I$, 比较拟合指数; $p$, 显著性概率值; RMSEA, 渐进残差均方和平方根; SRMR, 标准均方根残差。

$A I C$, akaike information criterion; $C F I$, comparative fit index; $p$, probability of significance; $R M S E A$, root mean square error of approximation; SRMR, standardized root mean square residual. 


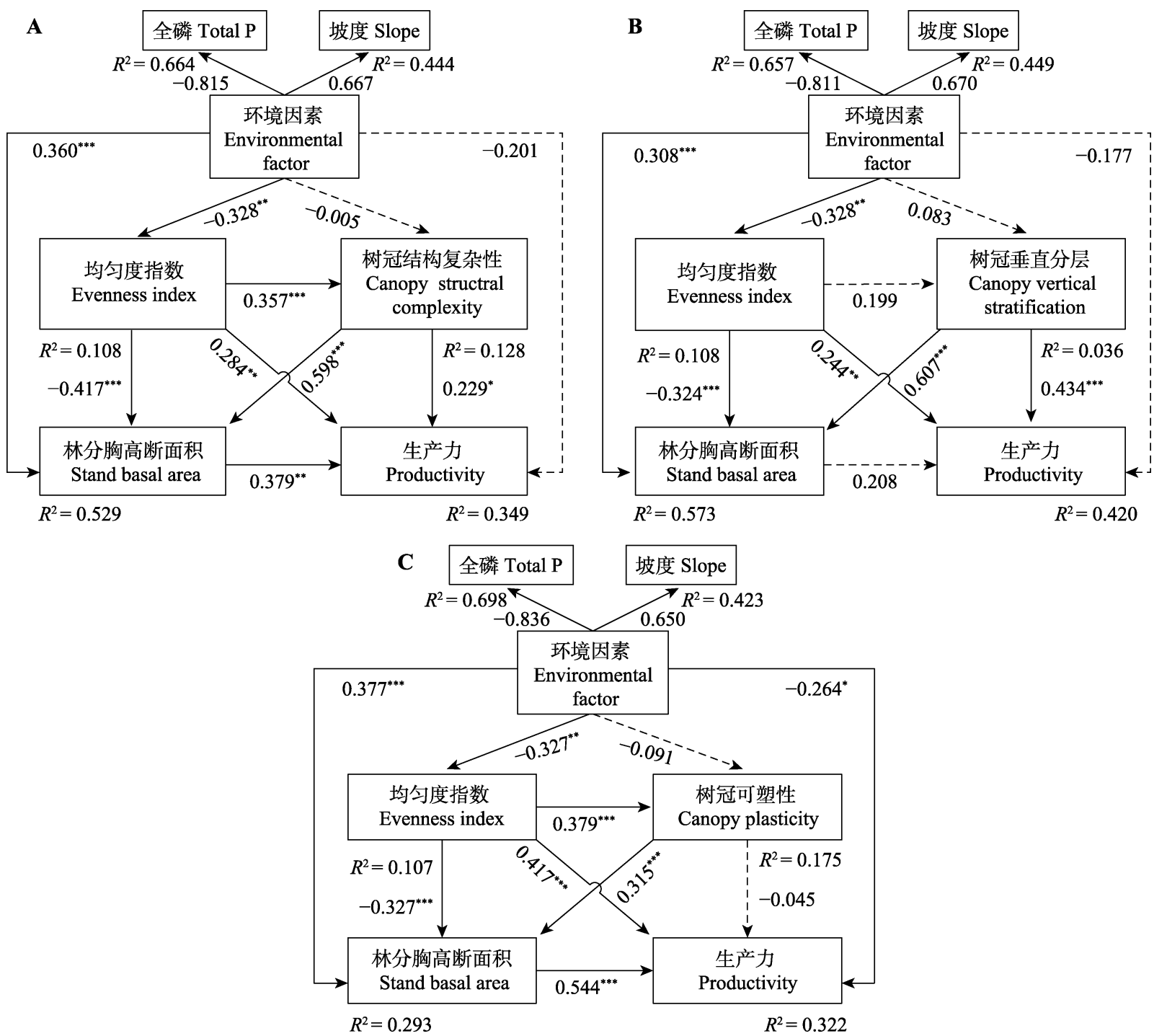

图3阔叶红松林树冠结构复杂性、均匀度指数、林分胸高断面积、环境因素(土壤全磷 $(\mathrm{P})$ 和坡度结合的潜变量)与生产力之 间的结构方程模型。A, 考虑树冠结构复杂性的模型。B, 考虑树冠垂直分层的模型。C, 考虑树冠可塑性的模型。图中实线 表示作用路径显著, 虚线表示作用路径不显著。箭头旁边的值是每个路径的标准化回归系数和显著性。 $R^{2}$ 表示由组合自变量 解释的因变量的总变化。*, $p<0.05 ; * *, p<0.01 ; * *, p<0.001$ 。

Fig. 3 Structural equation models (SEMs) for linking multivariate relationships among canopy structural complexity, evenness index, stand basal area, environmental factor (a latent variable of soil total phosphorus (P) and slope), and productivity in typical mixed broadleaved-Korean pine forest. A, Considering canopy structural complexity. B, Considering canopy vertical stratification. C, Considering canopy plasticity in the structural equation model. Solid lines indicate significant paths, and dash lines indicate insignificant paths. Standardized regression coefficients and significance are shown next to the arrow for each path. $R^{2}$ indicates the total variation in a dependent variable explained by the combined independent variables. ${ }^{*}, p<0.05 ; * *, p<0.01 ; * * *, p<0.001$.

接地作用于阔叶红松林生产力; 树冠可塑性对生产 力无显著影响 $(p>0.05)$, 仅通过影响林分胸高断面 积间接作用于生产力, 间接效应为 $0.171(p=$ 0.005)。然而, 仅考虑两者关系时, 树冠可塑性与生 产力显著正相关 $(p=0.018)$ (图4), 表明结合其他生 物和非生物因素后，树冠可塑性对生产力的作用被 减弱。物种多样性对树冠可塑性的直接效应为 0.379 $(p<0.001)$, 但通过树冠结构作用于生产力的间接 影响不显著 $(p>0.05)($ 图3C; 表6)。

\section{3 讨论}

\section{1 树冠结构复杂性和物种多样性对生产力的影响}

以往研究多样性-生产力关系时, 多集中于单 因素对生产力的影响，即仅考虑物种多样性或树冠 结构复杂性对生态系统功能的影响(Liang et al., 2016)。本研究以结构方程模型为手段，同时考虑了 以均匀度指数为代表的物种多样性, 以树冠镶嵌指 数观测值、预测值及其差值为代表的树冠结构复杂

www.plant-ecology.com 
表4 均匀度指数、树冠结构复杂性、林分胸高断面积和环境因素对典型阔叶红松林生产力的直接、间接和总效应

Table 4 Direct, indirect, and total standardized effects of evenness index (EI), canopy structural complexity (CSC), stand basal area (SBA) and environmental factor on productivity in a typical mixed broadleaved-Korean pine forest

\begin{tabular}{|c|c|c|c|c|}
\hline $\begin{array}{l}\text { 预测因子 } \\
\text { Predictor }\end{array}$ & $\begin{array}{c}\text { 直接效应 } \\
\text { Direct effect }\end{array}$ & $\begin{array}{l}\text { 间接效应途径 } \\
\text { Pathways of indirect effect }\end{array}$ & $\begin{array}{c}\text { 间接效应 } \\
\text { Indirect effect }\end{array}$ & $\begin{array}{c}\text { 总效应 } \\
\text { Total effect }\end{array}$ \\
\hline \multirow[t]{4}{*}{ 环境因子 Environmental factor } & $-0.201(p>0.05)$ & 总计 Summed & $0.042(p>0.05)$ & $-0.159(p>0.05)$ \\
\hline & & 通过均匀度指数 $\mathrm{Via} E I$ & $-0.093(p>0.05)$ & \\
\hline & & 通过树冠结构复杂性 Via CSC & $-0.001(p>0.05)$ & \\
\hline & & 通过林分胸高断面积 Via $S B A$ & $0.137(p=0.021)$ & \\
\hline \multirow[t]{3}{*}{ 均匀度指数 $E I$} & $0.284(p=0.006)$ & 总计 Summed & $-0.077(p>0.05)$ & $0.207(p=0.036)$ \\
\hline & & 通过树冠结构复杂性 Via CSC & $0.082(p>0.05)$ & \\
\hline & & 通过林分胸高断面积 Via $S B A$ & $-0.158(p=0.008)$ & \\
\hline \multirow[t]{2}{*}{ 树冠结构复杂性 CSC } & $0.229(p=0.045)$ & 总计 Summed & $0.227(p=0.004)$ & $0.455(p<0.001)$ \\
\hline & & 通过林分胸高断面积 Via $S B A$ & $0.227(p=0.004)$ & \\
\hline 林分胸高断面积 $S B A$ & $0.379(p=0.002)$ & & & $0.379(p=0.002)$ \\
\hline
\end{tabular}

表5 均匀度指数、树冠垂直分层、林分胸高断面积和环境因素对典型阔叶红松林生产力的直接、间接和总效应

Table 5 Direct, indirect, and total standardized effects of evenness index (EI), canopy vertical stratification $(C V S)$, stand basal area $(S B A)$ and environmental factor on productivity in a typical mixed broadleaved-Korean pine forest

\begin{tabular}{|c|c|c|c|c|}
\hline $\begin{array}{l}\text { 预测因子 } \\
\text { Predictor }\end{array}$ & $\begin{array}{c}\text { 直接效应 } \\
\text { Direct effect }\end{array}$ & $\begin{array}{l}\text { 间接效应途径 } \\
\text { Pathways of indirect effect }\end{array}$ & $\begin{array}{c}\text { 间接效应 } \\
\text { Indirect effect }\end{array}$ & $\begin{array}{c}\text { 总效应 } \\
\text { Total effect }\end{array}$ \\
\hline \multirow[t]{4}{*}{ 环境因子 Environmental factor } & $0.177(p>0.05)$ & 总计 Summed & $0.064(p>0.05)$ & $-0.146(p>0.05)$ \\
\hline & & 通过均匀度指数 Via $E I$ & $-0.080(p>0.05)$ & \\
\hline & & 通过树冠垂直分层 Via $C V S$ & $0.036(p>0.05)$ & \\
\hline & & 通过林分胸高断面积 Via $S B A$ & $0.137(p=0.021)$ & \\
\hline \multirow[t]{3}{*}{ 均匀度指数 $E I$} & $0.244(p=0.007)$ & 总计 Summed & $-0.067(p>0.05)$ & $0.263(p=0.006)$ \\
\hline & & 通过树冠垂直分层 Via $C V S$ & $0.020(p>0.05)$ & \\
\hline & & 通过林分胸高断面积 Via $S B A$ & $0.086(p>0.05)$ & \\
\hline \multirow[t]{2}{*}{ 树冠垂直分层 $C V S$} & $0.434(p<0.001)$ & 总计 Summed & $0.126(p>0.05)$ & $0.560(p<0.001)$ \\
\hline & & 通过林分胸高断面积 Via $S B A$ & $0.126(p>0.05)$ & \\
\hline 林分胸高断面积 $S B A$ & $0.208(p>0.05)$ & & & $0.208(p>0.05)$ \\
\hline
\end{tabular}

表6 均匀度指数、树冠可塑性、林分胸高断面积和环境因素对典型阔叶红松林生产力的直接、间接和总效应

Table 6 Direct, indirect, and total standardized effects of evenness index $(E I)$, canopy plasticity $(C P)$, stand basal area $(S B A)$ and environmental factors on productivity in a typical mixed broadleaved-Korean pine forest

\begin{tabular}{|c|c|c|c|c|}
\hline $\begin{array}{l}\text { 预测因子 } \\
\text { Predictor }\end{array}$ & $\begin{array}{c}\text { 直接效应 } \\
\text { Direct effect }\end{array}$ & $\begin{array}{l}\text { 间接效应途径 } \\
\text { Pathways of indirect effect }\end{array}$ & $\begin{array}{c}\text { 间接效应 } \\
\text { Indirect effect }\end{array}$ & $\begin{array}{c}\text { 总效应 } \\
\text { Total effect }\end{array}$ \\
\hline \multirow[t]{4}{*}{ 环境因子 Environmental factor } & $-0.264(p=0.024)$ & 总计 Summed & $0.073(p>0.05)$ & $-0.191(p>0.05)$ \\
\hline & & 通过均匀度指数 $\mathrm{Via} E I$ & $-0.136(p=0.011)$ & \\
\hline & & 通过树冠可塑性 Via $C P$ & $0.004(p>0.05)$ & \\
\hline & & 通过林分胸高断面积 Via $S B A$ & $0.205(p=0.006)$ & \\
\hline \multirow[t]{3}{*}{ 均匀度指数 $E I$} & $0.417(p<0.001)$ & 总计 Summed & $-0.195(p=0.014)$ & $0.222(p=0.030)$ \\
\hline & & 通过树冠可塑性 Via $C P$ & $-0.017(p>0.05)$ & \\
\hline & & 通过林分胸高断面积 Via $S B A$ & $-0.178(p=0.005)$ & \\
\hline \multirow[t]{2}{*}{ 树冠可塑性 $C P$} & $-0.045(p>0.05)$ & 总计 Summed & $0.171(p=0.005)$ & $0.126(p>0.05)$ \\
\hline & & 通过林分胸高断面积 Via $S B A$ & $0.171(p=0.005)$ & \\
\hline 林分胸高断面积 $S B A$ & $0.544(p<0.001)$ & & & $0.544(p<0.001)$ \\
\hline
\end{tabular}

性、树冠垂直分层和树冠可塑性, 以及林分胸高断 面积和环境因素对生产力的影响。

研究结果表明阔叶红松林物种多样性、树冠结
构复杂性和林分生产力之间存在显著的正相关关系 $(p<0.05)$ 。首先, 证实均匀度指数作为表征物种多 样性的重要指标, 与生产力密切相关 $(p=0.036)$ 。群 

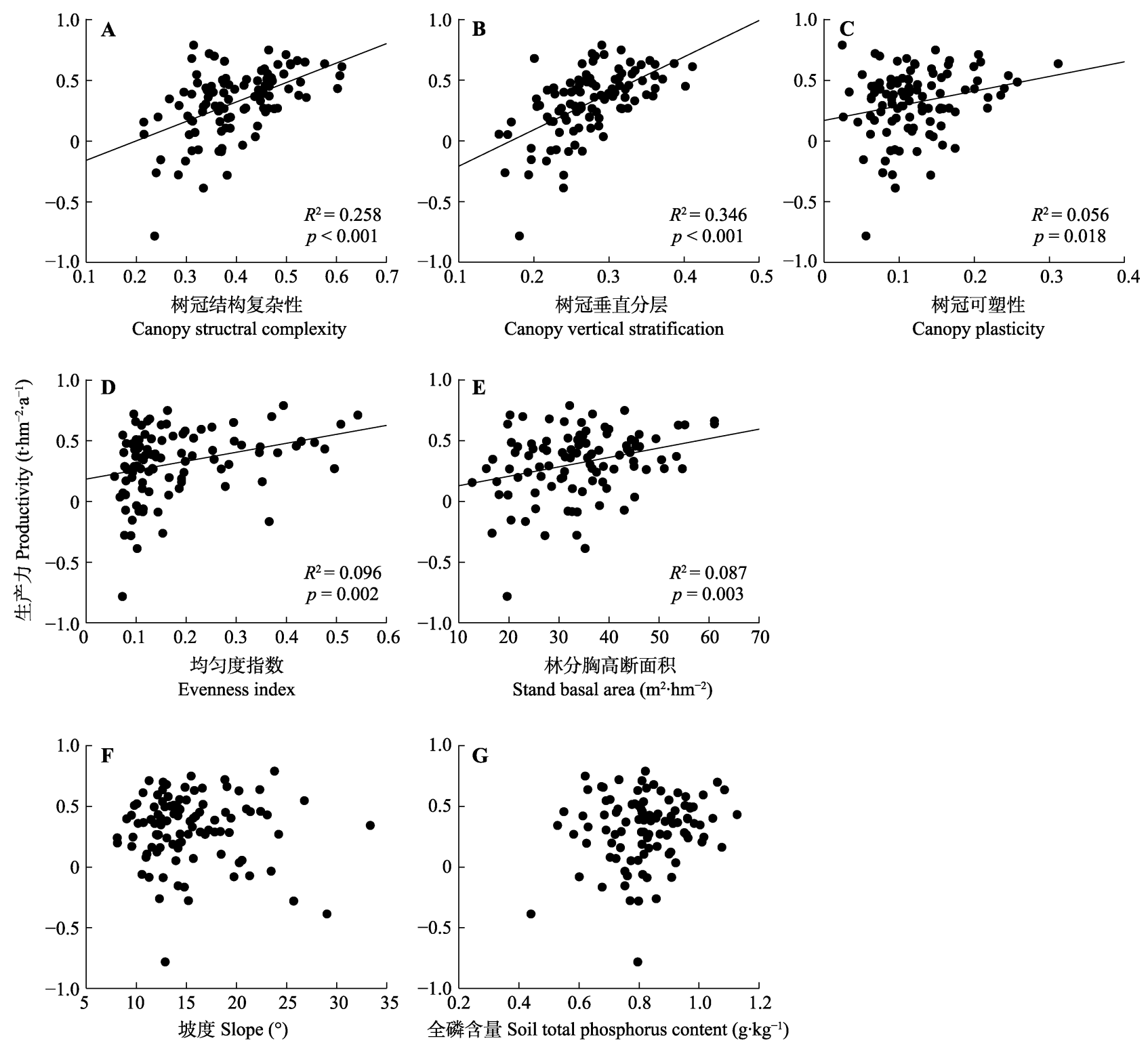

图4 典型阔叶红松林生产力(对数转换值)与各个解释变量的双变量关系。 $p<0.05$ 表示拟合关系显著; $p>0.05$ 时拟合关系不 显著, 无回归直线。

Fig. 4 Bivariate relationships between productivity (ln transformed) and explanatory variables in a typical mixed broadleavedKorean pine forest. Fitted regression is significant at $p<0.05$ and the relationships without fitted lines are insignificant at $p>0.05$.

落物种均匀度越高, 群落中各物种利用光照、水分 等资源更充分，具有更高的生产潜力(Hillerbrand et al., 2008)。其次, 研究发现了森林树冠结构复杂 性(包括水平与垂直方向)对生产力的重要作用 $(p<$ 0.001), 扩展了前人只重视单一多样性所展示的结 果(Zhang et al., 2012)。相较于物种多样性 $(p=0.036)$, 树冠结构复杂性更好地解释了该群落生产力的变异 $(p<0.001)$ (图3; 表4)。这可能是因为物种均匀度所 包含的关于物种的特征信息少(Dănescu et al., 2016; Bohn \& Huth, 2017), 而树冠结构复杂性是林分内 树木种内种间竞争的结果, 与树木生长直接相关
(Pretzsch, 2014)。林冠结构越复杂, 林冠中层和下层 的树木可通过冠层间隙获得的光资源越多, 形成单 位面积上更加密集的树冠，提高光利用率，从而促 进生产力(Bayer et al., 2013; Rissanen et al., 2019)。 但是, 也有研究表明在不同尺度中, 树冠结构复杂 性和物种多样性对生产力的影响存在差异, 且有关 树冠结构复杂性的研究较少, 还有待进一步研究 (Williams et al., 2017)。同时, 本研究未能验证物种 多样性通过树冠结构复杂性间接作用于生产力 $(p>$ $0.05)$, 出现不同结果的原因可能是因为森林类型的 不同或者在选取多样性指标上存在差异(Fahey et al., 
2015; Dănescu et al., 2016)。

本研究结果进一步表明, 树冠结构复杂性促进 生产力增长主要是树冠垂直分层的作用 $(p<0.001)$, 而树冠可塑性对生产力的作用并不显著 $(p>0.05)$ (图3; 表5, 表6)。作为反映树冠垂直方向分布的重 要指标, 树冠垂直分层对生产力的促进作用逐渐 受到研究人员的关注(Purves et al., 2007; Zheng et al., 2019)。此前, 多数研究将高大的乔木作为主林 层, 较矮的灌木作为亚林层, 比较不同林层植被对 群落生产力的贡献, 证实多林层的结构削弱了树 木对资源的竞争(尤其是对光的竞争), 提高了林分 生产力(Vockenhuber et al., 2011)。也有研究明确表 明, 树种的耐阴性是驱动群落生产力的重要因素, 具有不同生长潜力的落叶和常绿树种占据不同的 冠层位置, 延伸不同高度的树枝和叶片, 共同促进 物种共存与生物量积累(Toïgo et al., 2018)。本研究 结果同样表明, 不同树种因为自身生长特性, 分布 在不同冠层, 优势种红松特殊的圆锥形树冠, 可以 保证林下充足的光照, 伴生有紫椴、糠椴、五角枫 和冷杉等落叶和常绿树种, 不同树种对光照的分 层截留以及叶片对郁闭环境的生理适应驱动了树木 生长。这一结果有力地证明了树木对光线更全面的 多层利用促进了阔叶红松林生产力, 从而有助于我 们了解多层次森林群落的生态功能及其维持机制。

树冠可塑性表示树冠结构水平方向上分布的程 度, 是树木适应性生长的重要策略。Jucker等(2015) 发现, 可塑性较强的树冠结构才是群落生产力增长 的原因, 树冠垂直分层反而没有明显作用。而我们 的研究表明, 在结合了物种多样性、林分胸高断面 积和环境因素的作用后, 树冠可塑性与阔叶红松林 生产力之间不存在显著的相关关系 $(p>0.05)$, 这与 北温带森林的研究结果一致, 该研究发现处于演替 中后期的混交林的生产力几乎没有随着树冠可塑性 的变化而变化, 而其同一地区的幼林的树冠可塑性 与生产力之间存在较强的相关关系(Fahey et al., 2015)。演替前期的森林, 其树冠可塑性可以理解为 幼树为了获取光线分化成不同的形状, 而处于演替 后期的老龄林, 其树冠可塑性表现为大径级树木透 光率的增高(即叶片分布稀疏), 或者产生较大的林 隙, 反而没有明显的生物量积累(Seidel et al., 2011; Lin et al., 2014)。本研究中, 树冠可塑性的比例较小, 树木更倾向于投入高生长来捕捉光线, 同时表现出
较小的树冠横向发展幅度。考虑到许多冠层结构特 征的自相关性, 需要进一步探究其垂直和水平方向 上的功能角色(Hardiman et al., 2013)。

\section{2 其他生物和非生物因素对生产力的影响}

探讨非生物(地形和土壤理化性质)与生物(物种 多样性、树冠结构复杂性和林分结构)因素之间的 多元关系对理解生态系统功能具有重要意义(Yuan et al., 2018)。作为反映环境因素的两个重要指标, 土壤理化性质和地形对物种分布、树冠结构以及生 产力均有显著的影响 $(p<0.05)$ (Grace et al., 2016; Ali et al., 2019)。相较于以往只考虑其中一方的影响, 本研究采用结构方程模型的方法, 探讨了以坡度和 土壤全磷含量所代表的环境因素与生产力的关系, 并且进一步考虑了环境因素对树冠结构复杂性、物 种多样性和林分胸高断面积的影响, 结果表明在考 虑不同树冠结构复杂性的模型中, 环境因素的作用 存在差异, 仅考虑树冠可塑性的模型中对生产力有 显著的直接影响 $(p=0.024)$, 且通过林分胸高断面 积间接地作用于生产力 $(p=0.006)$, 这表明当树冠 结构与生产力关系相对较弱时, 环境因素是影响生 产力的重要因素; 而在其他两个模型中, 环境因素 对生产力的作用甚微 $(p>0.05)$, 可能是因为阔叶红 松林样地海拔位于425-509 m, 地形间差异相对较 小, 树种间的适应性生长更有助于解释生产力的变 化(Zhu et al., 2017; 温佩颖和金光泽, 2019)。此外, 环境因素对冠层的作用均不显著 $(p>0.05)$, 这一结 果同美国东部阔叶林的研究结果相同, 其树冠结构 复杂性的变异是由物种组成和树木大小所驱动, 未 受到干扰事件或者样地环境因素的影响(Hardiman et al., 2013)。具体环境因素对生产力的影响仍需要进 一步研究, 尤其是其在不同空间尺度和林分演替阶 段中的作用。

本研究证实林分胸高断面积是解释生产力变化 的重要因素, 林分胸高断面积自身可以促进生产力, 另外物种均匀度、树冠结构复杂性以及环境因素也 会通过影响林分胸高断面积, 而间接地影响生产 力。我们的结果也有助于解释Muth和Bazzaz (2003) 的发现, 即在研究单木树冠分布时, 胸高断面积是 最佳的预测因子。树木的树冠倾向于避开与大径木、 郁闭木和耐阴木相邻(Dănescu et al., 2016), 减少竞 争, 从而提高森林生产力。 


\section{4 结论}

本研究分析了阔叶红松林生产力与林分胸高断 面积、树冠结构复杂性、物种多样性和环境因素之 间的相关关系。结构方程模型分析结果表明, 树冠 结构复杂性、林分胸高断面积和物种多样性均与阔 叶红松林生产力呈显著正相关关系 $(p<0.05)$, 总体 效应依次降低, 而环境因素对生产力的作用不显著 $(p>0.05)$; 树冠结构复杂性中树冠垂直分层与生产 力呈显著正相关关系 $(p<0.001)$, 而树冠可塑性与 生产力无显著关系 $(p>0.05)$; 仅考虑树冠可塑性作 用时, 物种多样性和林分胸高断面积对生产力变化 的解释权重较大 $(p<0.05)$, 且移除树冠垂直分层的 作用后, 环境因素与生产力呈显著负相关关系 $(p=$ 0.024); 树冠垂直结构互补性和对光线更全面的多 层利用促进了阔叶红松林生产力, 以上结果为更加 深入理解温带老龄林生产力形成格局及其维持机制 提供了科学依据。

\section{参考文献}

Ali A, Lin SL, He JK, Kong FM, Yu JH, Jiang HS (2019). Tree crown complementarity links positive functional diversity and aboveground biomass along large-scale ecological gradients in tropical forests. Science of the Total Environment, 656, 45-54.

Ali A, Yan ER, Chen HYH, Chang SX, Zhao YT, Yang XD, Xu MS (2016). Stand structural diversity rather than species diversity enhances aboveground carbon storage in secondary subtropical forests in Eastern China. Biogeosciences, 13, 4627-4635.

Bartoń K (2016). Multi-model inference. Sociological Methods and Research, 33, 261-304.

Bayer D, Seifert S, Pretzsch H (2013). Structural crown properties of Norway spruce (Picea abies L. Karst.) and European beech (Fagus sylvatica L.) in mixed versus pure stands revealed by terrestrial laser scanning. Trees, 27, 10351047.

Bohn FJ, Huth A (2017). The importance of forest structure to biodiversity-productivity relationships. Royal Society Open Science, 4, 160521. DOI: 10.1098/rsos.160521.

Chave J, Condit R, Lao S, Caspersen JP, Foster RB, Hubbell SP (2003). Spatial and temporal variation of biomass in a tropical forest: results from a large census plot in Panama. Journal of Ecology, 91, 240-252.

Chen CG, Zhu JF (1989). A Handbook for Main Tree Species Biomass in Northeast China. China Forestry Press, Beijing. [陈传国, 朱俊凤 (1989). 东北主要林木生物量手册. 中国林业出版社，北京.]
Dănescu A, Albrecht AT, Bauhus J (2016). Structural diversity promotes productivity of mixed, uneven-aged forests in southwestern Germany. Oecologia, 182, 319-333.

Fahey RT, Fotis AT, Woods KD (2015). Quantifying canopy complexity and effects on productivity and resilience in late-successional hemlock-hardwood forests. Ecological Applications, 25, 834-847.

Forrester DI (2014). The spatial and temporal dynamics of species interactions in mixed-species forests: from pattern to process. Forest Ecology and Management, 312, 282-292.

Fotis AT, Morin TH, Fahey RT, Hardiman BS, Bohrer G, Curtis PS (2018). Forest structure in space and time: biotic and abiotic determinants of canopy complexity and their effects on net primary productivity. Agricultural and Forest Meteorology, 250-251, 181-191.

Grace JB, Anderson TM, Seabloom EW, Borer ET, Adler PB, Harpole WS, Hautier Y, Hillebrand H, Lind EM, Pärtel M, Bakker JD, Buckley YM, Crawley MJ, Damschen EI, Davies KF, et al. (2016). Integrative modelling reveals mechanisms linking productivity and plant species richness. Nature, 529, 390-393.

Guo YL, Wang B, Xiang WS, Ding T, Lu SH, Huang FZ, Wen SJ, Li DX, He YL, Li XK (2016). Responses of spatial pattern of woody plants' basal area to topographic factors in a tropical karst seasonal rainforest in Nonggang, Guangxi, southern China. Biodiversity Science, 24, 30-39. [郭屹立, 王斌, 向悟生, 丁涛, 陆树华, 黄甫昭, 文淑均, 李冬 兴, 何运林, 李先琨 (2016). 喀斯特季节性雨林木本植 物胸高断面积分布格局及其对地形因子的响应. 生物 多样性, 24, 30-39.]

Hardiman BS, Gough CM, Halperin A, Hofmeister KL, Nave LE, Bohrer G, Curtis PS (2013). Maintaining high rates of carbon storage in old forests: a mechanism linking canopy structure to forest function. Forest Ecology and Management, 298, 111-119.

Hillebrand H, Bennett DM, Cadotte MW (2008). Consequences of dominance: a review of evenness effects on local and regional ecosystem processes. Ecology, 89, 1510-1520.

Howard AL (2013). Handbook of structural equation modeling. Structural Equation Modeling: a Multidisciplinary Journal, 20, 354-360.

Jucker T, Bouriaud O, Coomes DA (2015). Crown plasticity enables trees to optimize canopy packing in mixed-species forests. Functional Ecology, 29, 1078-1086.

Klute A (1986). Methods of Soil Analysis: Part 1-Physical and Mineralogical Methods. 2nd ed. American Society of Agronomy, Soil Science Society of America, Madison, USA.

Legendre P, Mi XC, Ren HB, Ma KP, Yu MJ, Sun IF, He FL (2009). Partitioning beta diversity in a subtropical broadleaved forest of China. Ecology, 90, 663-674.

Liang J, Crowther TW, Picard N, Wiser S, Zhou M, Alberti G,

www.plant-ecology.com 
Schulze E-D, David McGuire A, Bozzato F, Pretzsch H, de Miguel S, Paquette A, Hérault B, Scherer-Lorenzen M, Barrett CB, et al. (2016). Positive biodiversity-productivity relationship predominant in global forests. Science, 354, aaf8957. DOI: 10.1126/science.aaf8957.

Lin F, Comita LS, Wang XG, Bai XJ, Yuan ZQ, Xing DL, Hao ZQ (2014). The contribution of understory light availability and biotic neighborhood to seedling survival in secondary versus old-growth temperate forest. Plant Ecology, 215, 795-807.

Muth CC, Bazzaz FA (2003). Tree canopy displacement and neighborhood interactions. Canadian Journal of Forest Research, 33, 1323-1330.

Pretzsch H (2014). Canopy space filling and tree crown morphology in mixed-species stands compared with monocultures. Forest Ecology and Management, 327, 251-264.

Purves DW, Lichstein JW, Pacala SW (2007). Crown plasticity and competition for canopy space: a new spatially implicit model parameterized for 250 North American tree species. PLOS ONE, 2, e870. DOI: 10.1371/journal.pone.0000870.

Quesada CA, Phillips OL, Schwarz M, Czimczik CI, Baker TR, Patiño S, Fyllas NM, Hodnett MG, Herrera R, Almeida S, Alvarez Dávila E, Arneth A, Arroyo L, Chao KJ, Dezzeo $\mathrm{N}$, et al. (2012). Basin-wide variations in Amazon forest structure and function are mediated by both soils and climate. Biogeosciences, 9, 2203-2246.

Rissanen K, Martin-Guay MO, Riopel-Bouvier AS, Paquette A (2019). Light interception in experimental forests affected by tree diversity and structural complexity of dominant canopy. Agricultural and Forest Meteorology, 278, 107655. DOI: 10.1016/j.agrformet.2019.107655.

Rosseel Y (2012). lavaan: an R package for structural equation modeling. Journal of Statistical Software, 48, 2. DOI: 10. 18637/jss.v048.i02.

Sapijanskas J, Paquette A, Potvin C, Kunert N, Loreau M (2014). Tropical tree diversity enhances light capture through crown plasticity and spatial and temporal niche differences. Ecology, 95, 2479-2492.

Seidel D, Leuschner C, Müller A, Krause B (2011). Crown plasticity in mixed forests - Quantifying asymmetry as a measure of competition using terrestrial laser scanning. Forest Ecology and Management, 261, 2123-2132.

Sterba H, Dirnberger G, Ritter T (2019). Vertical distribution of leaf area of European larch (Larix decidua Mill.) and Norway spruce (Picea abies L. Karst.) in pure and mixed stand. Forests, 10, 570. DOI: 10.3390/f10070570.

Toïgo M, Perot T, Courbaud B, Castagneyrol B, Gégout JC, Longuetaud F, Jactel H, Vallet P (2018). Difference in shade tolerance drives the mixture effect on oak productivity. Journal of Ecology, 106, 1073-1082.
Vockenhuber EA, Scherber C, Langenbruch C, Meißner M, Seidel D, Tscharntke T (2011). Tree diversity and environmental context predict herb species richness and cover in Germany's largest connected deciduous forest. Perspectives in Plant Ecology, Evolution and Systematics, 13, 111-119.

Wang YQ (1995). The Mixed Broadleaved-Korean Pine Forest. Northeast Forestry University Press, Harbin. [王业鿪 (1995). 阔叶红松林. 东北林业大学出版社, 哈尔滨.]

Wen PY, Jin GZ (2019). Effects of topography on species diversity in a typical mixed broadleaved-Korean pine forest. Acta Ecologica Sinica, 39, 945-956. [温佩颖, 金光泽 (2019). 地形对阔叶红松林物种多样性的影响. 生态学 报, 39, 945-956.]

Williams LJ, Paquette A, Cavender-Bares J, Messier C, Reich PB (2017). Spatial complementarity in tree crowns explains overyielding in species mixtures. Nature Ecology \& Evolution, 1, 0063. DOI: 10.1038/s41559-016-0063.

$\mathrm{Xu}$ LN, Jin GZ (2012). Species composition and community structure of a typical mixed broadleaved-Korean pine (Pinus koraiensis) forest plot in Liangshui Nature Reserve, Northeast China. Biodiversity Science, 20, 470-481. [徐丽 娜, 金光泽 (2012). 小兴安岭凉水典型阔叶红松林动态 监测样地: 物种组成与群落结构. 生物多样性, 20 , 470-481.]

Yuan Z, Ali A, Wang S, Gazol A, Freckleton R, Wang X, Lin F, Ye J, Zhou L, Hao Z, Loreau M (2018). Abiotic and biotic determinants of coarse woody productivity in temperate mixed forests. Science of the Total Environment, 630, 422-431.

Zeller L, Liang J, Pretzsch H (2018). Tree species richness enhances stand productivity while stand structure can have opposite effects, based on forest inventory data from Germany and the United States of America. Forest Ecosystems, 5, 4. DOI: 10.1186/s40663-017-0127-6.

Zhang Y, Chen HYH, Reich PB (2012). Forest productivity increases with evenness, species richness and trait variation: a global meta-analysis. Journal of Ecology, 100, 742749.

Zheng LT, Chen HYH, Yan ER (2019). Tree species diversity promotes litterfall productivity through crown complementarity in subtropical forests. Journal of Ecology, 107, 1852-1861.

Zhu Y, Cai HY, Jiang F, Jin GZ (2017). Variation of the biotic neighbourhood and topographic effects on tree survival in an old-growth temperate forest. Journal of Vegetation Science, 28, 1166-1177.

责任编委: 段昌群 编辑: 赵 航 
50 植物生态学报 Chinese Journal of Plant Ecology 2021, 45 (1): 38-50

附录I 不同尺度下生产力最优结构方程模型拟合结果

Supplement I Results of the best-fit structural equation models (SEMs) of productivity at varying scales

\begin{tabular}{lccccc}
\hline 尺度 Scale & 比较拟合指数 $C F I$ & 标准均方根残差 $S R M R$ & 渐进残差均方和平方根 $R M S E A$ & 赤池信息准则 $A I C$ & $p$ \\
\hline $10 \mathrm{~m} \times 10 \mathrm{~m}$ & 1 & 0.006 & 0 & 12400.938 & 0.653 \\
$20 \mathrm{~m} \times 20 \mathrm{~m}$ & 0.99 & 0.026 & 0.065 & 3105.839 & 0.118 \\
$30 \mathrm{~m} \times 30 \mathrm{~m}$ & 1 & 0.020 & 0 & 1308.250 & 0.720 \\
\hline
\end{tabular}

$A I C$, akaike information criterion; $C F I$, comparative fit index; RMSEA, root mean square error of approximation; $S R M R$, standardized root mean square residual.

附录II 典型阔叶红松林与生产力建立多重回归模型中各变量的相对重要性结果

Supplement II Results of the relative importance of each variable in multiple regression model with productivity in typical mixed broadleaved-Korean pine forest

\begin{tabular}{|c|c|c|}
\hline 解释变量 Explanatory variable & 变量重要性 Variable importance & 含变量模型 Containing model \\
\hline 均匀度指数 Evenness index & 0.65 & 5 \\
\hline Simpson 多样性指数 Simpson diversity index & 0.39 & 3 \\
\hline Shannon 多样性指数 Shannon diversity index & 0.28 & 2 \\
\hline 物种丰富度指数 Species richness index & 0.19 & 2 \\
\hline 坡度 Slope & 0.81 & 4 \\
\hline 海拔 Elevation & 0.56 & 3 \\
\hline 坡向 Aspect (sin) & 0.41 & 2 \\
\hline 坡向 Aspect (cos) & 0.16 & 1 \\
\hline 土壤全磷含量 Soil total phosphorus content & 1.00 & 8 \\
\hline 土壤速效氮含量 Soil available nitrogen content & 1.00 & 8 \\
\hline 土壤速效磷含量 Soil available phosphorus content & 1.00 & 8 \\
\hline 体积含水率 Soil volumetric moisture content & 0.91 & 7 \\
\hline 土壤容重 Soil bulk density & 0.49 & 4 \\
\hline 土壤速效钾含量 Soil available potassium content & 0.22 & 2 \\
\hline 质量含水率 Soil mass moisture content & 0.11 & 1 \\
\hline 土壤全氮含量 Soil total nitrogen content & 0.10 & 1 \\
\hline
\end{tabular}

